\title{
Endovascular Embolization of Fistulous Component of Gamma Knife-treated Arteriovenous Malformation in a Patient Presenting with Delayed Hemorrhage
}

\author{
Ajay Kumar ${ }^{1}$, Vikas Bhatia ${ }^{2}$, N Khandelwal ${ }^{3}$, Sunil K Gupta ${ }^{4}$
}

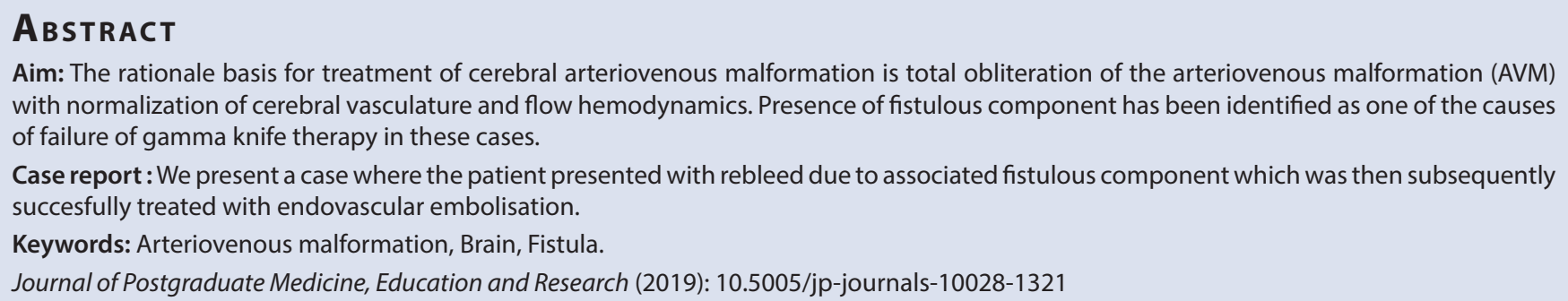

\section{INTRODUCTION}

$T^{\text {he }}$ he rational basis for the treatment of cerebral arteriovenous malformation is total obliteration of the AVM with normalization of cerebral vasculature and flow hemodynamics. Surgery, endovascular embolization, and radiosurgery as gamma knife are the therapeutic modalities currently employed for treatment of these AVM. Gamma knife radiosurgery is effective, with a cure rate of $60-85 \%$ at 2 years. ${ }^{1,2}$

Multiple factors have been implicated in treatment failure after Gamma knife. These patients have similar survival rates as untreated patients however there is a risk of repeat hemorrhage. ${ }^{3}$ One of the causative factors responsible for treatment failure is associated fistula component which shows poor response to Gamma knife therapy. ${ }^{4}$

We present a case of cerebral AVM who was treated with gamma radiosurgery, however, presented after 3 years with bleed due to associated AFV which was successfully managed with coil embolization. This fistulous component was not identified on initial DSA and became obvious after reduction of AVM nidus by gamma radiosurgery.

\section{Case presentation}

A 42-year female presented with a chief complaint of nonspecific headache for which she underwent a magnetic resonance imaging (MRI) scan which revealed evidence of AVM in the right frontal region. No prior history of any seizure, focal deficit or neurological dysfunction was seen. The patient was subsequently taken up for diagnostic subtraction angiography (DSA). There was evidence of AVM with nidus size of $5.4 \times 4.2 \mathrm{~cm}$ seen in the right frontal region with prominent feeders from right orbitofrontal and prefrontal branches of right MCA and orbitofrontal, frontopolar and anterior internal frontal branches of right ACA. No evidence of any intranidal or flow related aneurysm was seen. Early prominent draining veins were seen draining into the superior sagittal sinus and transverse sinus (Fig. 1).

Based on these findings patient was subsequently taken up for gamma knife radiosurgery. The patient received fractionated dose over of $18 \mathrm{~Gy}$ at the center and $9 \mathrm{~Gy}$ at the periphery with a
${ }^{1,3,4}$ Professor, ${ }^{2}$ Assistant Professor

${ }^{1-3}$ Department of Radiodiagnosis and Imaging, Postgraduate Institute of Medical Education and Research, Chandigarh, India

${ }^{4}$ Department of Neurosurgery, Postgraduate Institute of Medical Education and Research, Chandigarh, India

Corresponding Author: Vikas Bhatia, Assistant Professor, Department of Radiodiagnosis and Imaging, Postgraduate Institute of Medical Education and Research, Chandigarh, India, e-mail: drvikasbhatia@ gmail.com

How to cite this article: Kumar A, Bhatia V, Khandelwal N, Gupta SK. Endovascular Embolization of a Fistulous Component of Gamma Knifetreated Arteriovenous Malformation in Patient Presenting with Delayed Hemorrhage. J Postgrad Med Edu Res 2019;53(2):91-93.

Source of support: Nil

Conflict of interest: None

total irradiated volume of $33.4 \mathrm{~mL}$. The dose fractionated gamma knife radiosurgery (DFGKRS) schedule for the patient was for 3 consecutive days with a dose of $9 \mathrm{~Gy}$ at $50 \%$. After gamma knife therapy patient was followed up at every 6 months with clinical and magnetic resonance imaging (MRI) examinations.

There was no fresh symptoms and shrinkage of AVM nidus on two years follow-up. After 2.5 years of follow-up, this patient presented with a severe headache and altered sensorium. Fresh computed tomography $(C T)$ head (Fig. 1A) revealed evidence of right frontal bleed. The patient was subsequently taken for DSA examination which revealed a marked reduction in nidus size as compared to baseline DSA. However, there was evidence of high flow fistulous component seen between a prefrontal branch of right MCA and draining into the right transverse sinus (Figs $1 C$ and $D$ ). Few draining veins from the residual nidus were seen into the superior sagittal sinus. There was also ectasia of the distal right ICA and M1 MCA.

This AVF was not identified on previous DSA even on retrospective analysis as it was central in location and masked by the surrounding flow AVM nidus.

The patient was subsequently taken up for endovascular treatment, and successful embolization was performed using 2

() The Author(s). 2019 Open Access This article is distributed under the terms of the Creative Commons Attribution 4.0 International License (https://creativecommons. org/licenses/by-nc/4.0/), which permits unrestricted use, distribution, and non-commercial reproduction in any medium, provided you give appropriate credit to the original author(s) and the source, provide a link to the Creative Commons license, and indicate if changes were made. The Creative Commons Public Domain Dedication waiver (http://creativecommons.org/publicdomain/zero/1.0/) applies to the data made available in this article, unless otherwise stated. 

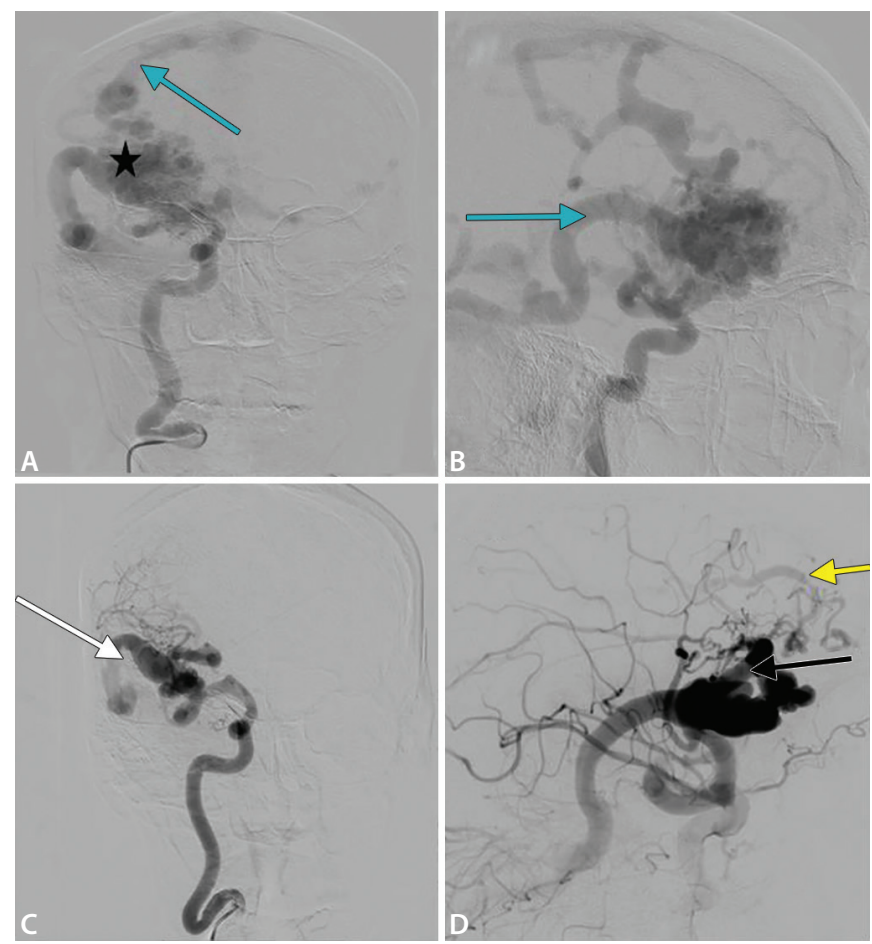

Figs 1 A to D: (A and B) Initial DSA with right ICA run AP and lateral view demonstrating $A V M$ in right frontal region (star) with prominent feeders from right MCA and ACA. No evidence of any intranidal or flow related aneurysm seen. Early prominent draining veins were seen draining into superior sagittal sinus and transverse sinus (blue arrows): (C and D) DSA with right ICA run AP and lateral view showing a high flow fistulous component between prefrontal branch of right MCA (black arrow) and draining into right transverse sinus (white arrow). There was marked resolution of AVM components with few veins draining into SSS(yellow arrow).

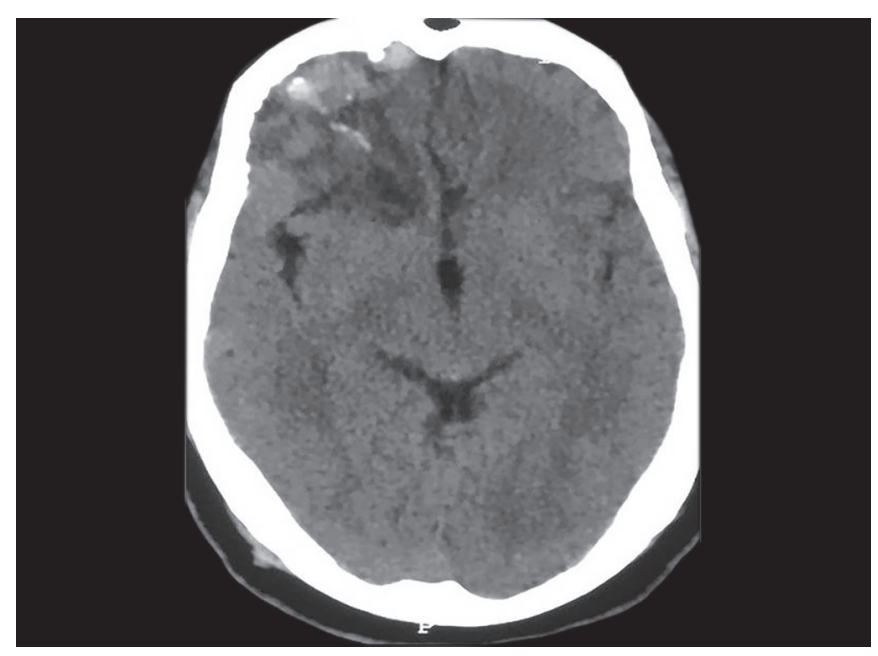

Fig. 3: NCCT head of the patient revealing hyperdense right frontal bleed

micro coils with the help of microcatheter resulting in complete obliteration of AVF (Figs $2 A$ and $B$ ).

Follow up DSA after 3 months revealed complete obliteration of AVF with a resolution of the ecstatic component of distal ICA and M1 MCA (Fig. 3).
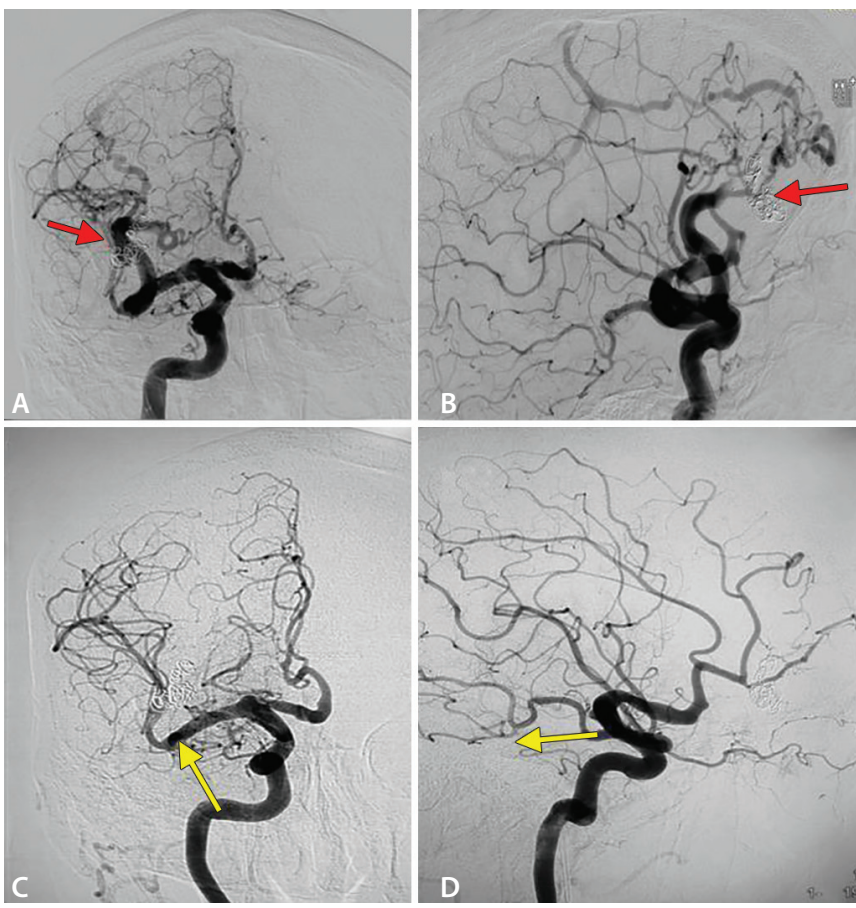

Figs $2 A$ to $D$ : (A and $B$ ) DSA with right ICA run AP and lateral view showing almost complete obliteration of AVF with 2 coils (red arrow). Early prominent draining veins were seen draining into superior sagittal sinus and transverse sinus (white arrows): (C and D) Check DSA 3 months after embolization showing with right ICA run AP and lateral views showing complete obliteration of AVF with coils in situ. Obliteration of the draining veins into superior sagittal sinus was seen with normalization of the caliber of right ICA and MCA (yellow arrow)

\section{Discussion}

Gamma knife surgery is a less invasive modality for the treatment of AVM with the good outcome however failed response is seen in about $30 \%$ of patients. Mukherjee et al. have shown in their study that DFGKRS on a daily schedule in cases of large AVM have shown excellent obliteration rates with acceptable toxicity. ${ }^{5}$ A similar protocol was used in our patient, and there was marked a reduction in the size of nidus as compared to baseline. The patient however presented with rebleed due to associated AVF which was unmasked as nidus was obliterated.

Multiple factors which have been reported to be associated with treatment failure with gamma knife include inadequate nidus demonstration, a large volume of the nidus, inadequate radiation dose and presence of arterio-venous fistulous component. ${ }^{3,6}$

The association of high-flow AVF has been associated with failure of Gamma knife treatment in approximately $5.8 \%$ of patients with AVM. ${ }^{3}$

An AVF is direct communication between an arterial feeder and venous drain without any intervening nidus. These have been categorized based on flow as low- or a high-flow and based on location into central and peripheral types. ${ }^{7}$ Central type is more prevalent and overlaps with a nidus of the AVM. These are frequently missed as they are difficult to demonstrate before gamma knife because of overlapping with arterial feeders, nidus, or venous channels. Few points on DSA can suggest the presence of AVF which include a demonstration of the prominent venous pouch in the nidus part with earlier opacification than other enlarged draining 
veins from the nidus. In our case, the central type of AVF was present which was masked by the presence of large nidus. Enlarged draining veins were seen into the superior sagittal sinus and right transverse sinus which had shown a similar rate of opacification. Thus AVF component could not be well demonstrated even on retrospect meticulous examination of diagnostic DSA.

The peripheral type of AVF is usually detected on DSA.,

The AVFs cause steal phenomenon with cerebral hypoperfusion and increased flow in cerebral veins leading to venous congestion, ischemia, and hemorrhage. ${ }^{7}$

Endovascular embolization has been used as an adjunct therapy for the management of cerebral AVMs. Pre-gamma surgery embolization has advantages to prevent the risk of hemorrhage during the latency and to increase chances of successful treatment of AVMs. Endovascular management of AVF components of AVM is controversial. Embolization to occlude AVF before gamma knife may decrease the hemodynamic venous stress with increase chances of success of the gamma radiosurgery. ${ }^{7}$ However, AVF itself may have a protective effect, and abrupt obliteration of AVF components may result in increased pressure gradient with resultant risk of nidus rupture. Thus, if embolization is done prior to radiosurgery both the fistula and maximum possible nidus part of AVM should be occluded. The major advantage of embolization of the AVF after gamma surgery is that the hemodynamic and angioarchitecture of the fistula can be clearly defined as much of the nidus is obliterated and complete cure can be achieved. It was also seen in our case as the fistulous component was seen after the gamma knife. ${ }^{8}$

Detailed DSA analysis of the angioarchitecture of the AVM is important for the assessment of response to gamma surgery which includes size, location, presence of an associated intranidal aneurysm, and AVF. Association of high flow fistulas is seen to respond less to gamma knife. Microcatheter super selective angiography has been shown to help in earlier detection of AVF. Pollock et al. ${ }^{9}$ used microcatheter super selective angiography as part of the pre-therapeutic evaluation to detect AVF. It can also help in the detection of intranidal aneurysms and immediate treatment in the same angiographic session. However, it is timeconsuming and expensive as compared to regular diagnostic DSA.

Studies with both pre-gamma knife and post gamma knife embolization have been reported in the literature. Chao-Bao Luo et al. identified 523 AVM patients which were treated with gamma knife at their institution, out of which 10 patients were identified with fistulous components and pre-gamma knife embolization was done. Successful embolization was done in nine patients using liquid adhesives ( 3 patients) and detachable coils (6 patients). No procedure-related complication was seen in their cases. CAVMs were completely obliterated subsequently with gamma knife in six patients, partial occlusion in one, while three were still followed for a response during their follow-up. ${ }^{7}$

In another study by the same author, they identified 19 patients with 21 AVFs undergoing embolization after stereotactic surgery.
They found complete fistula occlusion was achieved in all patients and residual AVMs were totally obliterated by embolization or additional radiosurgery in 12 patients. They concluded that most AVFs became evident after the obliteration of the nidus due to gamma knife and endovascular embolization effectively treats these fistulous components. ${ }^{8}$

Embolic agent selection for fistula closure is dependent on the morphology and flow of AVF. In high-flow fistulas associated with the venous pouch detachable coil is preferred due to precise control whereas in fistulas with tortuous and smaller feeders liquid embolic such as glue or onyx can be used. ${ }^{10,11}$

To conclude, we have demonstrated that a combined gamma knife and endovascular embolization is required to completely treat AVM with fistulous components. It is of paramount importance to identify these fistulas in initial angiography; however, they may still be obscured by the AVM nidus.

Endovascular embolization can successfully treat these fistulas and aids in a complete cure.

\section{References}

1. Steiner L, Lindquist C, Adler JR, et al. Clinical outcome of radiosurgery for cerebral arteriovenous malformations. J Neurosurg 1992;77:1-8.

2. Lunsford LD, Kondziolka Jl, Marks MP, et al. Stereotactic radiosurgery for arteriovenous malformations of the brain. J Neurosurg 1991;75:512-514.

3. Gallina P, Merienne L, Meder JF, et al. Failure in radiosurgery treatment of cerebral arteriovenous malformation. Neurosurgery 1998;42: 996-1004

4. Luo CB, Guo WY, Teng MM, et al. Embolization of arteriovenous fistula after radiosurgery for multiple cerebral arteriovenous malformations. Kaohsiung J Med Sci 2005;21:571e7

5. Mukherjee KK, Kumar N, Tripathi M, et al. Dose fractionated gamma knife radiosurgery for large arteriovenous malformations on daily or alternate day schedule outside the linear quadratic model: Proof of concept and early results. A substitute to volume fractionation. Neurol India 2017;65:826-835

6. Pollock BE, Kondziolka D, Lunsford LD, et al. Repeated stereotactic radiosurgery of arteriovenous malformations: factors associated with incomplete obliteration. Neurosurgery 1996;38:318e24

7. Luo CB, Guo WY, Teng MMH, et al. Fistula components of brain arteriovenous malformations: angioarchitecture analysis and embolisation prior to Gamma-Knife surgery. J Chin Med Assoc 2013;76:277-281

8. Luo CB, Guo WY, Teng MM, et al. Embolisation of arteriovenous fistula after radiosurgery for multiple cerebral arteriovenous malformations. Kaohsiung J Med Sci 2005;21:571-577

9. Pollock BE, Kondziolka D, Lunsford LD, et al. Repeat stereotactic radiosurgery of arteriovenous malformations: factors associated with incomplete obliteration. Neurosurgery 1996;38:318-324.

10. Luo CB, Teng MMH, Chang FC, et al. Endovascular treatment of intracranial high-flow arteriovenous fistulas by Guglielmi detachable coils. J Chin Med Assoc 2006;69:80e5.

11. Panagiotopoulos V, Gizewski E, Asgari S, et al. Embolization of intracranial arteriovenous malformations with ethylene-vinyl alcohol copolymer (Onyx). AJNR Am J Neuroradiol 2009;30:99-106. 